\title{
Correction to: Development of a novel strategy for fungal transformation based on a mutant locus conferring carboxin-resistance in Magnaporthe oryzae
}

Min Guo ${ }^{1 *+}$, Xiaolei Zhu ${ }^{1+}$, Hongxia $\mathrm{Li}^{2+}$, Leyong $\operatorname{Tan}^{1}$ and Yuemin Pan ${ }^{1 *}$

\section{Correction to: AMB Expr (2016) 6:57}

https://doi.org/10.1186/s13568-016-0232-x

Following publication of the original article (Guo et al. 2016), the authors of Guo et al. (2016) would like to make

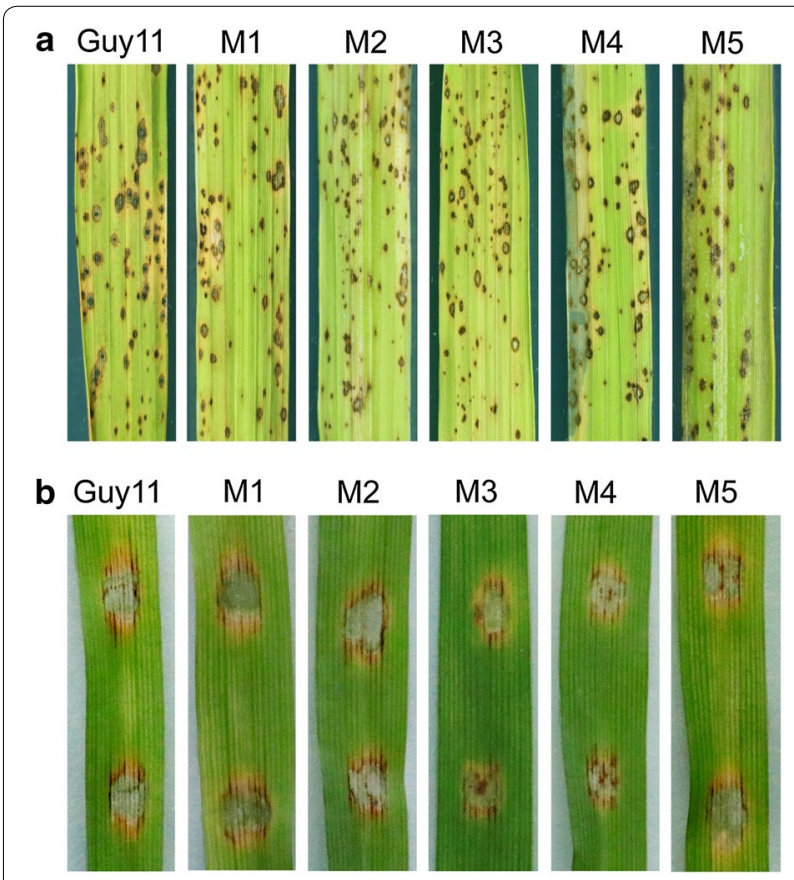

Fig. 4 Pathogenic assay for Mosdi $1^{\text {ReGFP }}$ mutants. The pathogenicity was tested for both wild type Guy11 and Mosdi RR RFP mutants, and no significant differences were identified among the strains. a Disease symptoms caused by strains on 14-day-old rice seedlings. b Disease symptoms caused by strains on 7-day-old barley leaves a correction for a figure in their published article. An image in Fig. 4b (Lane 4, M3) were mistakenly used for strain M3. We checked the original images and replaced it with the correct Fig. 4b as showed below. We confirm that this change do not alter the findings of this work.

\section{Author details}

${ }^{1}$ Department of Plant Pathology, College of Plant Protection, Anhui Agricultural University, Hefei 230036, China. ${ }^{2}$ Anhui Research Institute of Chemical Industry, Hefei 230041, China.

The original article can be found online at https://doi.org/10.1186/s1356 8-016-0232-x

Received: 17 June 2019 Accepted: 17 June 2019

Published online: 28 June 2019

\section{Reference}

Guo M, Zhu X, Li H, Tan L, Pan Y (2016) Development of a novel strategy for fungal transformation based on a mutant locus conferring carboxinresistance in Magnaporthe oryzae. AMB Expr 6:57. https://doi.org/10.1186/ s13568-016-0232-x

\section{Publisher's Note}

Springer Nature remains neutral with regard to jurisdictional claims in published maps and institutional affiliations.

*Correspondence: kandylemon@163.com; panyuemin2008@163.com

${ }^{\dagger}$ Min Guo, Xiaolei Zhu and Hongxia Li contributed equally to this work

1 Department of Plant Pathology, College of Plant Protection, Anhui

Agricultural University, Hefei 230036, China

Full list of author information is available at the end of the article 